\title{
Author self-citation in medical literature
}

\author{
Nataša Kovačić, Aleksandra Mišak
}

See related article page 1925

$\mathrm{I}$ $\mathrm{n}$ this issue Gami and colleagues ${ }^{1}$ report on their investigation of author self-citation (the practice of citing one's previous publications in a new publication) as a possible source of bias in bibliometric assessment of the importance of a journal or an author. Focusing on articles about diabetes mellitus as representative of the general clinical medical literature, they found that nearly one-fifth of all citations per year were author self-citations. They found no association between methodologic quality of articles and frequency of author self-citation. Compared with review articles, original articles had double the proportion of author self-citations. Articles published in highly cited journals had a smaller proportion of author self-citations than articles published in less-cited journals.

Citing is an established way for authors to declare their sources of information and politely recognize someone's intellectual property. The importance of an article, author or journal can be estimated through the number of citations each acquires. For journals, such a number is transformed into the impact factor, which approximates the frequency with which articles have been cited in the 2 years after publication. ${ }^{2}$ However imperfect and potentially unfair it may be, the impact factor is the best measure of a journal's quality we have today. ${ }^{3,4}$ But what can the proportion of a certain type of citation, such as author self-citation, tell us about a given article, author or journal?

A high rate of author self-citation may result from the fact that authors stick to their specific field of research and, naturally, rely on their previous results. To reduce article length, authors may cite previous work in which, for example, the same methods are described. It is also possible, however, that they overestimate the importance of their earlier research compared with other work they could have cited, or that they want to increase artificially the number of citations to their own work, thus distorting the perception of its importance. Institutional criteria for academic advancement take into account the total number of publications by the author and sometimes the total number of citations of that author's work. The latter might provide incentive for authors to self-cite, but self-citation does not really reflect the visibility and quality of their work. Finally, authors who publish a lot have more opportunities to cite their own previous work, which is why author self-citation rates may correlate with authors' publishing productivity. For these reasons, it is impossible to determine the level of authors' integrity with regard to self-citation.

Gami and colleagues' finding that review articles had a smaller proportion of self-citations than original scientific articles $^{1}$ may be explained by the fact that review articles are more widely read and therefore receive more citations in general.

Both journal self-citation (when articles in a journal cite previous articles in the same journal) and author self-citation may influence the journal's impact factor. ${ }^{5}$ From the e-table presented by Gami and colleagues ${ }^{1}$ it is clear that author self-citations accounted for a negligible proportion of citations in the high-impact journals and, therefore, would not substantially distort the impact factor of those journals. From the same data, one can also presume that there is an inverse correlation between the proportion of author self-citations and the impact factor of a journal. Our analysis of citations of the Croatian Medical Journal indicated that an increase in the absolute number of citations was indeed followed by a decrease in the proportion of both author and journal self-citations. ${ }^{6}$ The decrease in author self-citations could have been due to widening of the author pool (a result of better international visibility of the journal) or to increased quality of articles (which would attract independent citations), or it may simply have been mathematical, self-citations having been "diluted" by the higher number of independent citations. The decrease in the proportion of journal self-citations probably reflected the journal's increased visibility. ${ }^{6}$ Although a high proportion of journal self-citations may indeed increase the impact factor of a journal, in small journals this increase is illusory: it does not reflect an increase in international visibility, since the flow of information is limited mostly to the pool of the journal's authors and readers.

Whatever the reason, a high proportion of self-citations per article cannot be taken solely as a reflection of the limited quality of a journal: some journals have a narrower scope, either thematically or geographically, and consequently a smaller pool of authors. Journals that are both influential and important are characterized by a high impact factor, the publication of "milestone" articles and prestigious authors. Journals that make an important but less influential contribution typically publish in a fruitful area of research occupied by fewer investigators who are nonetheless highly productive and well cited. Journals with limited influence and importance publish in a highly specific area, have a small circulation, a high degree of overlap between the readership and the author pool, and receive 
few citations. For almost identical reasons, an article or author with a high proportion of self-citations cannot be accused of lower research quality or integrity. This is supported by Gami and colleagues' finding that "self-citations had little relation with the quality of an article."' The total number of citations also has to be taken into account. A highly cited article, author or journal with a substantial proportion of self-citations (considering the total citation number) is more visible than the rarely cited without any self-citations.

In this way, this noteworthy article ${ }^{1}$ opens the question of the "morally allowable" proportion of self-citations and offers an elegant and workable model for the pertinent research.

From the Department of Anatomy (Kovačić) and the Croatian Medical Journal (both authors), Zagreb University School of Medicine, Zagreb, Croatia.

Competing interests: None declared.
Contributors: Both authors contributed substantially to all phases of manuscript preparation and approved the version to be published.

\section{References}

1. Gami AS, Montori VM, Wilczynski NL, Haynes RB. Author self-citation in the diabetes literature. CMA72004;170(13):1925-7.

2. Garfield E. Journal impact factor: a brief review [editorial]. CMAJ 1999;161: 979-80.

3. Christopher MM. The impact factor: getting a grip. Vet Clin Pathol 2003;32: 98-100.

4. Seglen PO. Why the impact factor of journals should not be used for evaluating research. BM7 1997;314:498-502.

5. Fassoulaki A, Paraskeva A, Papilas K, Karabinis G. Self-citations in six anaesthesia journals and their significance in determining the impact factor. $\mathrm{Br} \mathrm{J}$ Anaesth 2000;84:266-9.

6. Kovačić N, Mišak A. What can be learned from impact factor of Croatian Medical Journal, 1994-2003? Croat Med J 2004;45:18-24.

Correspondence to: Dr. Nataša Kovačić, Department of Anatomy, Zagreb University School of Medicine, Šalata 3B, 10000 Zagreb, Croatia; fax+385-1-4590-222; natasa@mef.hr

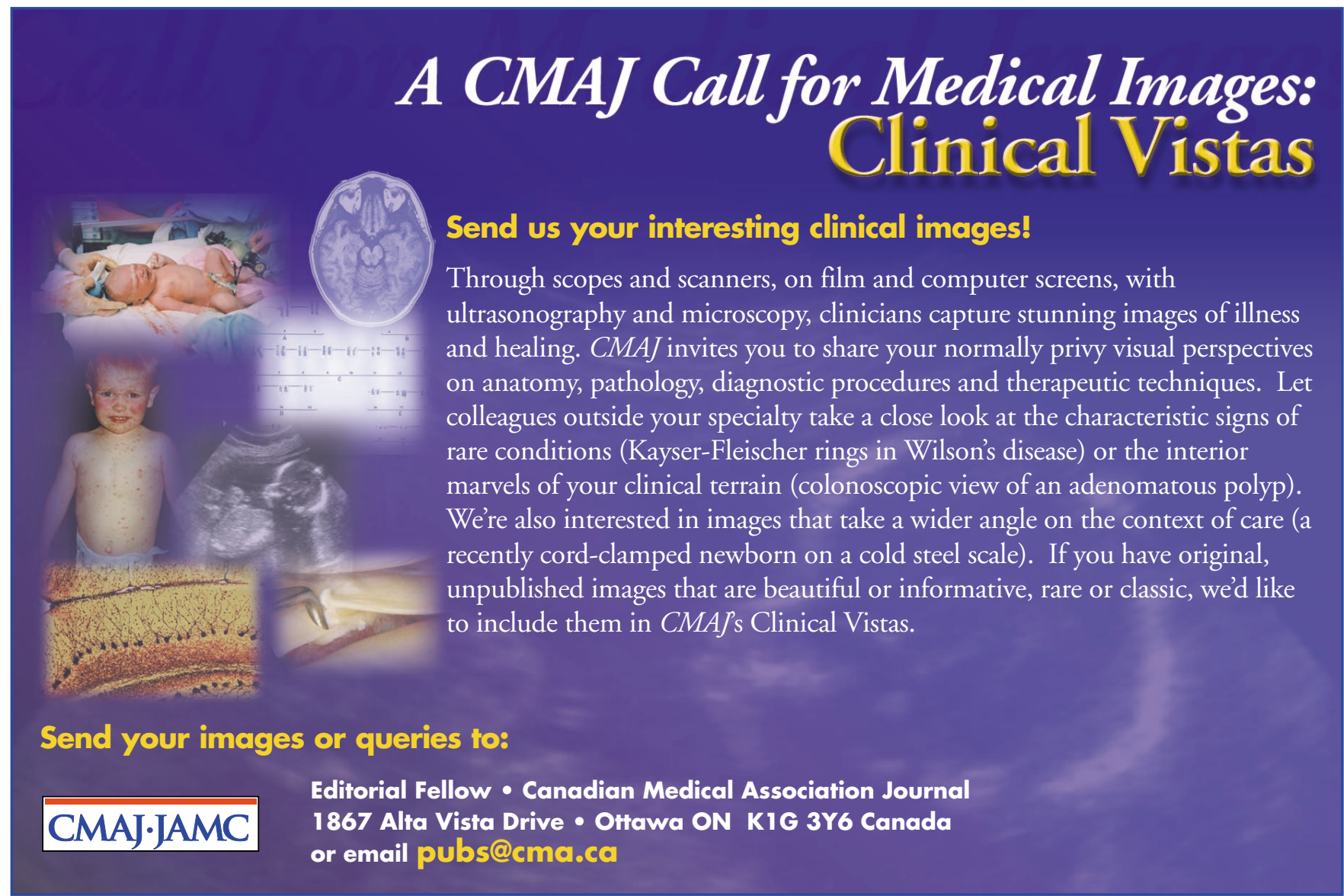

